\title{
Random matrix description of decaying quantum systems
}

\section{T Gorin ${ }^{1}$}

${ }^{1}$ Max-Planck-Institut für Physik komplexer Systeme, Nöthnitzer Str. 38, D-01187 Dresden, Germany

\begin{abstract}
This contribution describes a statistical model for decaying quantum systems (e.g. photo-dissociation or -ionization). It takes the interference between direct and indirect decay processes explicitely into account. The resulting expressions for the partial decay amplitudes and the corresponding cross sections may be considered a many-channel many-resonance generalization of Fano's original work on resonance lineshapes [Phys. Rev 124, 1866 (1961)].

A statistical (random matrix) model is then introduced. It allows to describe chaotic scattering systems with tunable couplings to the decay channels. We focus on the autocorrelation function of the total (photo) cross section, and we find that it depends on the same combination of parameters, as the Fano-parameter distribution. These combinations are statistical variants of the one-channel Fano parameter. It is thus possible to study Fano interference (i.e. the interference between direct and indirect decay paths) on the basis of the autocorrelation function, and thereby in the regime of overlapping resonances. It allows us, to study the Fano interference in the limit of strongly overlapping resonances, where we find a persisting effect on the level of the weak localization correction.
\end{abstract}

PACS numbers: 05.45.Mt, 03.65.Nk

Submitted to: J. Phys. A: Math. Gen.

E-mail: gorin@pks.mpg.de 


\section{Introduction}

Molecular photodissociation [1, 2, 3, 4, 5, 6] and atomic autoionization [7] are examples of quantum-mechanical decay processes: Within the dipole approximation, the absorption of a photon excites the quantum system into an energy region, which allows the dissociation and/or ionization of the system. Technically speaking, one observes the decay of an initial wave packet $|\alpha\rangle$ within a scattering system, via accessible open channels. Given a scattering system with Hamiltonian $H$, the partial decay amplitudes $t_{a}(E)$ are of central importance, as they define the partial and total (photo) cross sections:

$$
t_{a}(E)=\left\langle\Psi^{(a)}(E) \mid \alpha\right\rangle \quad \sigma_{a}(E)=\sigma_{0}\left|t_{a}(E)\right|^{2} \quad \sigma(E)=\sum_{c} \sigma_{c}(E) .
$$

Here, $\Psi^{(a)}(E)$ is the post-controlled scattering solution of the problem. This means that, asymptotically, there is one single outgoing plane wave in channel $a$. The sum in the definition of the total cross section $\sigma(E)$ runs over all open channels. The proportionality constant $\sigma_{0}$ depends on the excitation mechanism. As we will not discuss those mechanisms, we set $\sigma_{0}=1$. This description reduces the whole process to a half collision processes $[8,9,10]$. Note that we will mostly use the terminology from photo absorption, for simplicity, being well aware of the fact that other excitation mechanisms are equally possible.

We aim at a description of chaotic scattering systems, which have a large interaction region (in terms of elementary Planck cells), but a finite number of open channels. Such a situation occurs frequently in the photodissociation of simple molecules, where the potential surface allows trajectories coming from infinity to enter the interaction region only through so called "bottlenecks" (transition states). In this situation, scattering trajectories may have very short dwell times: They either do not find the bottleneck to enter the interaction region, or, due to symmetry, leave the interaction region after only a few bounces (Ehrenfest time). These are direct processes. The remaining trajectories "randomize" and leave the interaction region only after the average dwell time, which is proportional to the size of the openings. They contribute to the indirect decay.

In [11, 12], Alhassid and Fyodorov formulate and solve a statistical model for the total cross section. This quantity has the convenient property that it can be expressed in terms of the Greens function [13, 14]. With the help of Feshbach's projection formalism $[15,16]$, the full Hilbert space is divided into an orthogonal sum of a number of quasi bound states, and an $M$-channel continuum. In [12], an analytical expression for the autocorrelation function of $\sigma(E)$ is derived, by using the supersymmetry method. The result is quite similar to the "Verbaarschot-WeidenmüllerZirnbauer" (VWZ) integral [17].

In particular dynamical systems, deviations from the statistical model are quite possible. In the semiclassical regime, for instance, Policot-Ruelle resonances may give rise to characteristic signatures in the autocorrelation function of the total photo cross section $[18,19]$. A different source for deviations, is the interference with direct decay 
processes (Fano interference). In the limit of isolated resonances, it leads to asymmetric lineshapes [20, 21]. The statistical model of Alhassid and Fyodorov has been generalized recently, to include direct processes $[22,23]$. The basic formalism has been developed in nuclear reaction theory [24] (and references therein); see also reference [25].

Fano interference (Beutler-Fano lineshapes) has also been studied in mesoscopic transport. It is shown in reference [26] that Fano interference can be observed in the conductance of quantum dots coupled to single mode leads. Even though these results are not simply related to the present model, they still point at important applications. Quite generally, a Fano resonance may be used as a kind of built-in interferometer, which, for instance, is very sensitive to decoherence effects [27]. Also temperature effects as well as Coulomb interaction effects can be studied [28].

In this paper, we address the question how to detect and quantify Fano interference in the many channel case, and in the regime of overlapping resonances. For that purpose, we review in section 2 the statistical model for half collision processes, as developed in [22, 23]. The mapping formalism of reference [23], together with an important generalization is presented in section 3 . We then consider the limit of isolated resonances, where the resonance lineshapes can be described by a complex Fano parameter (section 4). The statistics of the Fano parameter has been considered in [29], for the one channel case. Here, we identify those quantities, which determine the Fano-parameter distribution in the many channel case. For each channel, one can find a statistical analog of the one-channel Fano parameter. In section 5, we turn to the study of the autocorrelation function. Surprisingly, it depends on very similar quantities. This allows to quantify Fano interference, beyond the regime of isolated resonances. In section 6, we study Fano interference in the "semiclassical regime", where many channels are strongly coupled to the interaction region. Even there, we find a noticeable effect. Its strength is comparable to the weak localization correction in mesoscopic transport [30]. A summary is given in section 7 .

In the present paper, the discussion is restricted to the case of orthogonal invariance, which allows to use the VWZ-integral [17] to compute autocorrelation functions. For broken time-reversal symmetry, the corresponding result for the S-matrix element correlations has been given recently [31]. It would allow to repeat the analysis of total cross section autocorrelation functions for that case.

\section{The model}

We follow the approach adopted in $[12,22,23]$ where resonant and direct processes are treated separately by means of the Feshbach approach $[15,16]$ : the Hilbert space of excited states is divided into a subspace of bound states ( $Q$-space), and its complement. The Schrödinger equation restricted to that complement is treated as an auxiliary scattering system. Choosing the bound space appropriately, it looses all resonant features. Its scattering solutions serve as a basis, in which the resonant scattering 
via the subspace of bound states is formulated.

We consider the scattering problem (or the half collision problem) in a small energy interval, away from any thresholds, such that the number of open channels, denoted by $M$, remains constant. Then, the partial decay amplitudes, defined in equation (1), may be written as

$t_{a}(E)=\left\langle a \mid \alpha^{\text {out }}\right\rangle+\left\langle a\left|V^{\dagger} \frac{1}{E-H_{\text {eff }}}\left(\left|\alpha^{\text {in }}\right\rangle-\mathrm{i} \pi V\left|\alpha^{\text {out }}\right\rangle\right) \quad H_{\text {eff }}=H_{0}-\mathrm{i} \pi V V^{\dagger}\right.\right.$,

where the vector $|\alpha\rangle$ has been decomposed $|\alpha\rangle=\left|\alpha^{\text {in }}\right\rangle \oplus\left|\alpha^{\text {out }}\right\rangle$ according to the partitioning of the Hilbert space, above. Here, $H_{0}$ is a $N \times N$-matrix of the original Hamiltonian projected onto $Q$-space. The matrix $V$ contains the transition amplitudes between the basis states in $Q$-space, and the scattering states $|c\rangle$. It has $M$ column vectors (the channel vectors), each of dimension $N$.

Note that $t_{a}(E)$ may be considered as a transition amplitude between the state $|\alpha\rangle$ and the scattering states $|a\rangle$ of the auxiliary resonance-free scattering system. These are typically not the free scattering solutions. To obtain the transition amplitudes into the free scattering states, one also needs the scattering matrix of the auxiliary scattering system. Below, we focus on the total photo cross section, where such considerations are of minor importance.

To obtain the total photo cross section, one may sum over all partial cross sections, or use a particular form of the optical theorem [13]. It relies on the fact that the scattering solutions $\left|\Psi^{(c)}(E)\right\rangle$ form a complete basis. Therefore:

$$
\begin{aligned}
\sigma(E) & =\sum_{c=1}^{M}\left\langle\alpha \mid \Psi^{(c)}\right\rangle\left\langle\Psi^{(c)}(E) \mid \alpha\right\rangle=\langle\alpha|\delta(E-H)| \alpha\rangle=\frac{1}{\pi} \operatorname{Im}\left\langle\alpha\left|G\left(E^{-}\right)\right| \alpha\right\rangle \\
& =\left\|\alpha^{\text {out }}\right\|^{2}-\pi^{-1} \operatorname{Im}\left[\left(\left|\alpha^{\text {in }}\right\rangle+\mathrm{i} \pi V\left|\alpha^{\text {out }}\right\rangle\right)^{\dagger} \frac{1}{E-H_{\text {eff }}}\left(\left|\alpha^{\text {in }}\right\rangle-\mathrm{i} \pi V\left|\alpha^{\text {out }}\right\rangle\right)\right] .
\end{aligned}
$$

Generally, we will assume that the column vectors of $V$ are pairwise orthogonal. If that is not the case, one may use a singular value decomposition [32]: $V=V_{0} s$, where $s$ is a unitary matrix. It allows to replace $V$ by $V_{0}$, whose column vectors are pairwise orthogonal. Due to $V\left|\alpha^{\text {out }}\right\rangle=V_{0}\left|\alpha_{s}^{\text {out }}\right\rangle$ with $\left|\alpha_{s}^{\text {out }}\right\rangle=s\left|\alpha^{\text {out }}\right\rangle$, the expression (3) remains unchanged except for a redefinition of the direct decay amplitudes.

\subsection{The statistical model}

For the derivation of the above expressions for $t_{a}(E)$ and $\sigma(E)$, we have used an arbitrary but fixed basis in $Q$-space. In that basis, we assume $V, \alpha^{\text {in }}$ and $\alpha^{\text {out }}$ to be fixed, and take $H_{0}$ from the Gaussian orthogonal ensemble (GOE). For the full collision problem, the statistical properties of the resulting S-matrix have been studied in detail $[17,33,34]$. Indirect photo decay has been studied in [12], and the effects of direct decay paths has been considered in $[22,23]$.

For the description of decay processes, we have $3 M+1$ independent parameters, which define the model: 
- The norm of the column vectors of $V$ measure the coupling strengths to the decay channels.

- The direct decay amplitudes $\alpha_{c}^{\text {out }}=\left\langle c \mid \alpha^{\text {out }}\right\rangle$ give the overlaps of the initial state with the scattering states of the auxiliary scattering system. They describe direct decay processes, which may may lead to Fano interference.

- The overlaps of $\alpha^{\text {in }}$ with the column vectors of $V$, i.e. $\left\langle c\left|V^{\dagger}\right| \alpha^{\text {in }}\right\rangle$, may give rise to Fano interference, also. As we will see, this type of interference is not equivalent to the one before.

- The norm of the component of $\left|\alpha^{\text {in }}\right\rangle$, which is orthogonal to all column vectors of $V$. That component is responsible for the indirect decay processes.

\section{The mapping formalism}

The mapping formalism has been introduced in [23]. It allows to express the partial decay amplitudes as well as the total photo cross section in terms of an extended scattering matrix. This is convenient if analytical results for the statistical scattering model are to be transfered to the present case. Here, we will use it to compute the average (this section) and the autocorrelation function (section 6) of the total photo cross section. In its original formulation, $\left|\alpha^{\text {in }}\right\rangle$ is required to be orthogonal to the column vectors of $V$. Here, the formalism is generalized, to allow for non-zero overlaps between $\left|\alpha^{\text {in }}\right\rangle$ and the channel vectors. This leads to the distinction between external and internal Fano interference.

Assume, the initial state $\left|\alpha^{\text {in }}\right\rangle$ has overlap with the channel vectors, and that channel vectors are coupled to the continuum with non-vanishing transmission coefficients. In that case, we can always find a $\left|\alpha^{\mathrm{io}}\right\rangle$, such that

$$
\left|\alpha^{\text {in }}\right\rangle=\left|\alpha_{0}^{\text {in }}\right\rangle+\pi V\left|\alpha^{\text {io }}\right\rangle
$$

where $\left|\alpha_{0}^{\text {in }}\right\rangle$ is orthogonal to all column vectors of $V$. Then, one can write for the transition amplitudes $t_{a}$ [equation (2)] and the total cross section [equation (3)]:

$t_{a}=\left\langle a \mid \alpha^{\text {out }}\right\rangle+\left\langle a\left|V^{\dagger} \frac{1}{E-H_{\text {eff }}}\left(\left|\alpha_{0}^{\text {in }}\right\rangle-\mathrm{i} \pi V\left|\alpha_{+}^{\text {out }}\right\rangle\right) \quad\right| \alpha_{ \pm}^{\text {out }}\right\rangle=\left|\alpha^{\text {out }}\right\rangle \pm \mathrm{i}\left|\alpha^{\text {io }}\right\rangle$
$\sigma(E)=\left\|\alpha^{\text {out }}\right\|^{2}-\pi^{-1} \operatorname{Im}\left[\left(\left|\alpha_{0}^{\text {in }}\right\rangle+\mathrm{i} \pi V\left|\alpha_{-}^{\text {out }}\right\rangle\right)^{\dagger} \frac{1}{E-H_{\text {eff }}}\left(\left|\alpha_{0}^{\text {in }}\right\rangle-\mathrm{i} \pi V\left|\alpha_{+}^{\text {out }}\right\rangle\right)\right]$.

Following [23], this suggests to introduce the following extended scattering matrix:

$S(E, \delta)=\mathbf{1}_{1+M}-2 \mathrm{i} \pi W^{\dagger} \frac{1}{E-F_{\text {eff }}} W \quad W=\left(\alpha_{0}^{\text {in }} \delta /(2 \pi), V\right)$,

which contains only the orthogonal part of $\left|\alpha^{\text {in }}\right\rangle$. Now, one has to find transformation matrices $u(\delta)$ and $v(\delta)$, such that $S^{\prime}(E, \delta)=u(\delta) S(E, \delta) v^{T}(\delta)$, may be used to construct the partial decay amplitudes, and the total photo cross section. One obtains the 
following answer:

$u(\delta)=\left(\begin{array}{c|c}\mathrm{i} & \left(\alpha_{-}^{\text {out }}\right)^{\dagger} \delta / 2 \\ \hline 0 & \\ \vdots & \mathbf{1}_{M} \\ 0 & \end{array}\right) \quad v(\delta)=\left(\begin{array}{c|c}\mathrm{i} & \left(\alpha_{+}^{\text {out }}\right)^{T} \delta / 2 \\ \hline 0 & \\ \vdots & \mathbf{1}_{M} \\ 0 & \end{array}\right)$.

The transformation of $S(E, \delta)$ is reminiscent of an "Engelbrecht-Weidenmüller" transformation [35], although for $\delta>0, u(\delta)$ and $v(\delta)$ are not unitary. The partial decay amplitudes and the total photo cross section can be obtained from:

$t_{a}(E)=\frac{\left\langle a \mid \alpha_{-}^{\text {out }}\right\rangle}{2}+\lim _{\delta \rightarrow 0} \delta^{-1} S_{a 0}^{\prime}(E, \delta)$

$\sigma(E)=\left\|\alpha^{\text {out }}\right\|^{2}-\frac{1}{2} \operatorname{Re}\left\langle\alpha_{-}^{\text {out }} \mid \alpha_{+}^{\text {out }}\right\rangle+\lim _{\delta \rightarrow 0} \frac{2}{\delta^{2}} \operatorname{Re}\left[1+S_{00}^{\prime}(E, \delta)\right]$.

The average total cross section As a first application, we will compute the average total cross section $\langle\sigma(E)\rangle$ in the center of the spectrum. To this end, we use the fact that the average extended S-matrix is given by [17]:

$\left\langle S_{a b}(0, \delta)\right\rangle=\delta_{a b} \frac{1-\lambda_{a}}{1+\lambda_{a}} \quad \lambda_{a}=\pi^{2} \rho_{0}\left\|\boldsymbol{v}_{a}\right\|^{2} \quad \lambda_{0}=\frac{\delta^{2}}{4} \rho_{0}\left\|\alpha_{0}^{\text {in }}\right\|^{2}$,

where for $a=1, \ldots, M, \boldsymbol{v}_{a}$ are the respective column vectors of the coupling matrix $V$. The level density is denoted by $\rho_{0}$, i.e. the average level spacing is $\Delta=1 /\left(N \rho_{0}\right)$. As the average S-matrix is diagonal, we obtain:

$$
\left\langle S_{00}^{\prime}\right\rangle=-\frac{1-\lambda_{0}}{1+\lambda_{0}}+\frac{\delta^{2}}{4}\left\langle\alpha_{-}^{\text {out }}|\bar{S}| \alpha_{+}^{\text {out }}\right\rangle,
$$

where $\bar{S}$ is the average of the original scattering matrix (of dimension $M$ ). From this it follows with equation (9):

$$
\langle\sigma\rangle=\left\|\alpha^{\text {out }}\right\|^{2}+\rho_{0}\left\|\alpha_{0}^{\text {in }}\right\|^{2}+\frac{1}{2} \operatorname{Re}\left\langle\alpha_{-}^{\text {out }}|(\bar{S}-1)| \alpha_{+}^{\text {out }}\right\rangle .
$$

Provided, the components of $\left|\alpha^{\text {out }}\right\rangle$ and $\left|\alpha^{\text {in }}\right\rangle$ are real, we may proceed a bit further. In equation (4), $\left|\alpha^{\text {io }}\right\rangle$ is chosen such that

$$
\left\langle c \mid \alpha^{\mathrm{in}}\right\rangle=\pi\left\langle\boldsymbol{v}_{c} \mid \alpha^{\mathrm{io}}\right\rangle=\pi\left\|\boldsymbol{v}_{c}\right\|\left\langle c \mid \alpha^{\mathrm{io}}\right\rangle \quad \Rightarrow \quad\left\langle c \mid \alpha^{\mathrm{io}}\right\rangle=\sqrt{\rho_{0} / \lambda_{c}}\left\langle c \mid \alpha^{\mathrm{in}}\right\rangle .
$$

Therefore:

$$
\begin{aligned}
\langle\sigma\rangle & =\left\|\alpha^{\text {out }}\right\|^{2}+\rho_{0}\left\|\alpha_{0}^{\text {in }}\right\|^{2}-\sum_{c}\left(\left|\alpha_{c}^{\text {out }}\right|^{2}-\frac{\rho_{0}}{\lambda_{c}}\left|\alpha_{c}^{\text {in }}\right|^{2}\right) \frac{\lambda_{c}}{1+\lambda_{c}} \\
& =\left\|\alpha^{\text {out }}\right\|^{2}+\rho_{0}\left\|\alpha^{\text {in }}\right\|^{2}-\sum_{c}\left(\left|\alpha_{c}^{\text {out }}\right|^{2}+\rho_{0}\left|\alpha_{c}^{\text {in }}\right|^{2}\right) \frac{\lambda_{c}}{1+\lambda_{c}} .
\end{aligned}
$$

On the level of the average total cross section, it makes no difference, whether $\alpha^{\text {in }}$ has overlap with the external channel region, or internal states (inside $Q$-space) which are connected to the channel region via transmission coefficients. For later use, we define the direct photo cross section as $\sigma_{\text {dir }}=\left\|\alpha^{\text {out }}\right\|^{2}$, and the indirect photo cross section as $\sigma_{\text {ind }}=\rho_{0}\left\|\alpha_{0}^{\text {in }}\right\|^{2}$. 


\section{Fano lineshapes: the limit of isolated resonances}

The effect of direct reaction paths is most evident in the limit of weak coupling, where individual resonances can be observed. In this regime, the resonances have asymmetric lineshapes (Beutler-Fano profiles) [20, 21], i.e. the cross section near resonance can be parametrised as

$$
\sigma(E)=\sigma_{\text {dir }} \frac{|\varepsilon+q|^{2}}{\varepsilon^{2}+1}=\sigma_{\text {dir }} \frac{\left(\varepsilon+q_{1}\right)^{2}+q_{2}^{2}}{\varepsilon^{2}+1} \quad \varepsilon=\frac{E-E_{j}}{\Gamma_{j} / 2} .
$$

While in the one channel case, the Fano parameter $q$ may be assumed real, one needs a complex Fano parameter $q=q_{1}+\mathrm{i} q_{2}$ in the case of many channels. The imaginary part of the Fano parameter lifts the resonance, such that the cross section at its minimum is no longer zero. As shown below, the background cross section (the cross section at large $|\varepsilon|)$ is $\sigma_{\text {dir }}=\left\|\alpha^{\text {out }}\right\|^{2}$, independent of the imaginary part of the Fano parameter.

In the limit of isolated resonances, the effective Hamiltonian $H_{\text {eff }}$ can be assumed diagonal in the eigenbasis of $H_{0}$. Using that basis in equation (3), we obtain:

$\sigma(E)=\left\|\alpha^{\text {out }}\right\|^{2}-\frac{1}{\pi} \sum_{j} \operatorname{Im}\left[\frac{\left(a_{j}+\mathrm{i} b_{j}\right)^{*}\left(a_{j}-\mathrm{i} b_{j}\right)}{E-E_{j}+\mathrm{i} \Gamma_{j} / 2}\right]$.

where $a_{j}=\left\langle j \mid \alpha^{\text {in }}\right\rangle, b_{j}=\pi \sum_{c} V_{j c} \alpha_{c}^{\text {out }}$ and $\Gamma_{j}=2 \pi \sum_{c} V_{j c}^{2}$. If these parameters may be assumed real (e.g. due to time reversal invariance), we obtain in the vicinity of the $j$ 'th resonance:

$$
\sigma(E) \approx \sigma_{j}(E)=\left\|\alpha^{\text {out }}\right\|^{2}-\frac{1}{\varepsilon^{2}+1} \frac{2}{\pi \Gamma_{j}} \operatorname{Im}\left[(\varepsilon-\mathrm{i})\left(a_{j}-\mathrm{i} b_{j}\right)^{2}\right] .
$$

This expression can be put into the form of equation (15) with $\sigma_{\text {dir }}=\left\|\alpha^{\text {out }}\right\|^{2}$. This yields the following values for the real and the imaginary part of the Fano parameter:

$$
q_{1}=\frac{a_{j} b_{j}}{D_{j}} \quad q_{2}=\sqrt{\left(1+\frac{a_{j}^{2}}{D_{j}}\right)\left(1-\frac{b_{j}^{2}}{D_{j}}\right)},
$$

where $D_{j}=\pi\left\|\alpha^{\text {out }}\right\|^{2} \Gamma_{j} / 2 \geq b_{j}^{2}$ due to the Schwarz inequality (it makes sure that $q_{2}$ is always real).

In the case of a regular cross section, it makes sense to study the individual resonances and their lineshapes. However, if the lineshape (i.e. the Fano parameter) fluctuates randomly from resonance to resonance, a statistical analysis is more appropriate. Let us rewrite equation (18) in terms of $M+1$ independent normalized Gaussian random variables. We will take into account that $\left|\alpha^{\text {in }}\right\rangle$ may have some overlap with channel vectors (the column vectors of $V$ ).

$$
\begin{aligned}
& V_{j c} \rightarrow s_{c} x_{c} \quad a_{j}=\left\langle j \mid \alpha_{0}^{\mathrm{in}}\right\rangle+\pi \sum_{c} V_{j c} \alpha_{c}^{\mathrm{io}} \rightarrow s_{0}\left(x_{0}+\pi \sum_{c} x_{c} \frac{s_{c} \alpha_{c}^{\mathrm{io}}}{s_{0}}\right) \\
& b_{j}=\pi \sum_{c} x_{c} \frac{s_{c} \alpha_{c}^{\text {out }}}{s_{0}} \quad D_{j}=\pi^{2} \frac{\left\|\alpha^{\text {out }}\right\|^{2}}{s_{0}^{2}} \sum_{c} x_{c}^{2} s_{c}^{2} .
\end{aligned}
$$

Besides $\sigma_{\text {dir }}=\left\|\alpha^{\text {out }}\right\|^{2}$ and $\sigma_{\text {ind }} \propto s_{0}^{2}$, the following parameter combinations can be identified: $s_{c}, s_{c} \alpha_{c}^{\text {out }} / s_{0}$, and $s_{c} \alpha_{c}^{\text {io }} / s_{0}$, where $c=1, \ldots, M$. As these parameters come 
along with centered Gaussian random variables, their sign is irrelevant. We thus prefer to consider their squares:

$s_{0}^{2}=\frac{\left\|\alpha_{0}^{\text {in }}\right\|^{2}}{N} \quad s_{c}^{2}=\left\langle V_{j c}^{2}\right\rangle \rightarrow \frac{1}{4 \pi^{2}} \frac{T_{c}}{N \rho_{0}}$

$\frac{s_{c}^{2}\left|\alpha_{c}^{\text {out }}\right|^{2}}{s_{0}^{2}} \rightarrow \frac{\left|\alpha_{c}^{\text {out }}\right|^{2} T_{c}}{4 \pi^{2} \sigma_{\text {ind }}}=\frac{\tau_{c}^{\text {out }}}{4 \pi^{2}} \quad \frac{s_{c}^{2}\left|\alpha_{c}^{\text {io }}\right|^{2}}{s_{0}^{2}} \rightarrow \frac{\left|\alpha_{c}^{\text {io }}\right|^{2} T_{c}}{4 \pi^{2} \sigma_{\text {ind }}}=\frac{\tau_{c}^{\text {io }}}{4 \pi^{2}}$.

As we will see below, the autocorrelation function depends almost on the same set of parameters. That means, an analysis of the autocorrelation function gives much the same information, with the advantage that its application is not restricted to the regime of isolated resonances.

One channel case In that case $b_{j}^{2}=D_{j}$ so that the Fano parameter becomes real:

$$
q_{1}=\frac{a_{j}}{\pi \alpha_{1}^{\text {out }} V_{j 1}}=\frac{\left\langle j \mid \alpha_{0}^{\text {in }}\right\rangle}{\pi \alpha_{1}^{\text {out }} V_{j 1}}+\frac{\alpha_{1}^{\text {io }}}{\alpha_{1}^{\text {out }}} \quad q_{2}=0,
$$

where we have again separated that part of $\left|\alpha^{\text {in }}\right\rangle$ which is orthogonal to $V$ and that which is parallel. For precisely that case, the distribution of $q_{1}$ has been computed in [29]. The result is a shifted Lorentzian with width $g$ determined by:

$$
g^{2}=\frac{\left\langle\left\langle j \mid \alpha_{0}^{\text {in }}\right\rangle^{2}\right\rangle}{\pi^{2}\left|\alpha^{\text {out }}\right|^{2}\left\langle V_{j 1}^{2}\right\rangle}=\frac{4 \sigma_{\text {ind }}}{\left|\alpha^{\text {out }}\right|^{2} T_{1}}=\frac{4}{\tau_{1}^{\text {out }}},
$$

whereas the shift is given by

$$
-\bar{q}=\frac{\alpha_{1}^{\text {io }}}{\alpha_{1}^{\text {out }}}=\frac{\tau_{1}^{\text {io }}}{\tau_{1}^{\text {out }}} .
$$

That part of the work in [29], which deals with time reversal invariant systems, is hence fully contained in the present model.

\section{Autocorrelation function of the total photo cross section}

In section 4, direct decay processes have been studied, as they affect the lineshapes of isolated resonances. Once the resonances start to overlap, such an analysis is no longer possible. Here, we study the interference effects on the autocorrelation function. This quantity has the advantage, that there are no restrictions on the coupling strengths to decay channels (transmission coefficients). This allows to study Fano interference even in the regime of strongly overlapping resonances (section 6).

Internal versus external Fano interference Here, we will study the autocorrelation function of the total photo cross section (3), with the help of the mapping formalism and the VWZ-integral [17]. The latter provides an analytical expression for the correlation function between two matrix elements of the (extended) scattering matrix in equation (6). With this, it is possible to compute correlation functions between partial decay amplitudes, as well as between total cross sections. It is also possible to choose 
different initial states $\alpha$, if that would be of interest. In the case of two total cross sections, we would consider the correlation function

$$
C\left[\sigma_{1}, \sigma_{2}\right](w)=\left\langle\sigma_{1}(E-w \Delta / 2) \sigma_{2}(E+w \Delta / 2)\right\rangle-\left\langle\sigma_{1}(E)\right\rangle\left\langle\sigma_{2}(E)\right\rangle,
$$

where $\langle\ldots\rangle$ denote a spectral ane/or ensemble average, as appropriate, and $\Delta$ the mean level (resonance) spacing. Here, $\sigma^{(1)}\left(\sigma^{(2)}\right)$ denotes the total photo cross section for the initial state $\left|\alpha_{1}\right\rangle\left(\left|\alpha_{2}\right\rangle\right)$. Usually, we find it more convenient to consider the correlation function in the time domain:

$$
\hat{C}\left[\sigma_{1}, \sigma_{2}\right](t)=\int \mathrm{d} w \mathrm{e}^{2 \pi \mathrm{i} w t} C\left[\sigma_{1}, \sigma_{2}\right](w) .
$$

Due to the fact that $C\left[\sigma_{1}, \sigma_{2}\right](w)$ is defined on the unfolded energy axis, $t$ measures time in units of the Heisenberg time.

For simplicity, and because this case is typically considered in the literature, the following discussion will be restricted to the autocorrelation function of the total photo cross section. Using for $\sigma$ the expression (9) from the mapping formalism, (section 3) one obtains:

$$
C[\sigma]=\lim _{\delta \rightarrow 0} \frac{1}{\delta^{4}} C\left[S_{00}^{\prime}+S_{00}^{\prime *}, S_{00}^{\prime}+S_{00}^{\prime *}\right]=2 \lim _{\delta \rightarrow 0} \frac{1}{\delta^{4}} \operatorname{Re} C\left[S_{00}^{\prime}, S_{00}^{\prime *}\right],
$$

and for its Fourier transform (see reference [36]):

$$
\hat{C}[\sigma]=\lim _{\delta \rightarrow 0} \frac{1}{\delta^{4}} \hat{C}\left[S_{00}^{\prime}, S_{00}^{* *}\right] .
$$

The auxiliary scattering matrix $S^{\prime}(E, \delta)$ is defined within the mapping formalism [see equations (6) and (7)]. For its matrix element $S_{00}^{\prime}$, one obtains:

$S_{00}^{\prime}=-S_{00}+\frac{\mathrm{i}}{2} \delta \sum_{a=1}^{M}\left[\left(\alpha_{-}^{\text {out }}\right)_{a}^{*} S_{a 0}+\left(\alpha_{+}^{\text {out }}\right)_{a} S_{0 a}\right]+\frac{\delta^{2}}{4} \sum_{a, b=1}^{M}\left(\alpha_{-}^{\text {out }}\right)_{a}^{*}\left(\alpha_{+}^{\text {out }}\right)_{b} S_{a b}$.

This gives $\hat{C}[\sigma](t)$ in terms of a linear combination of correlation functions between S-matrix elements, where the VWZ-integral [17] applies. Due to equation (A.1) many such correlation functions vanish. After a bit of algebra, one arrives at the following result:

$$
\begin{aligned}
\hat{C}[\sigma] & =\sigma_{\text {ind }}^{2} \mathcal{I}\left\{\left|2 \Delta_{0}-\frac{1}{2} \sum_{a=1}^{M} \tau_{a} \Delta_{a}\right|^{2}+4 \Pi_{00}+\frac{1}{2} \sum_{a=1}^{M}\left[\tau_{a}+\tau_{a}^{*}+\tau_{a}^{-}+\tau_{a}^{+}\right] \Pi_{0 a}\right. \\
& \left.+\frac{1}{8} \sum_{a, b=1}^{M}\left[\tau_{a}^{-} \tau_{b}^{+}+\tau_{a} \tau_{b}\right] \Pi_{a b}\right\} \\
\tau_{a}= & \left(\alpha_{-}^{\text {out }}\right)_{a}\left(\alpha_{+}^{\text {out }}\right)_{a}^{*} T_{a} / \sigma_{\text {ind }} \quad \tau_{a}^{ \pm}=\left|\left(\alpha_{ \pm}^{\text {out }}\right)_{a}\right|^{2} T_{a} / \sigma_{\text {ind }} .
\end{aligned}
$$

If the Fano interference is purely external, then $\tau_{a}=\tau_{a}^{ \pm}$: real, and we obtain the result from [23]:

$$
\hat{C}[\sigma](t)=\mathcal{I}\left\{\left(2 \Delta_{0}-\frac{1}{2} \sum_{a=1}^{M} \tau_{a} \Delta_{a}\right)^{2}+4 \Pi_{00}+2 \sum_{a=1}^{M} \tau_{a} \Pi_{0 a}+\frac{1}{4} \sum_{a, b=1}^{M} \tau_{a} \tau_{b} \Pi_{a b}\right\}
$$


where $\tau_{c}=\left|\alpha_{c}^{\text {out }}\right|^{2} T_{c} / \sigma_{\text {ind }}=\tau_{c}^{\text {out }}$ as given in equation (20). The parameters $\tau_{c}$ and $\tau_{c}^{ \pm}$ are the statistical variants of the Fano parameter; see section 4. Eventually, we call them "statistical Fano parameters" for short. If Fano interference is purely internal, then $\tau_{a}^{ \pm}=\left|\alpha_{a}^{\mathrm{io}}\right|^{2} T_{a} / \sigma_{\mathrm{ind}}=\tau_{a}^{\mathrm{io}}, \tau_{a}=-\tau_{a}^{ \pm}$. Therefore:

$$
\hat{C}[\sigma](t)=\mathcal{I}\left\{\left(2 \Delta_{0}+\frac{1}{2} \sum_{a=1}^{M} \tau_{a}^{ \pm} \Delta_{a}\right)^{2}+4 \Pi_{00}+\frac{1}{4} \sum_{a, b=1}^{M} \tau_{a}^{ \pm} \tau_{b}^{ \pm} \Pi_{a b}\right\} .
$$

\section{Limit of many open channels}

We consider two cases. The first is the absorptive limit. There, all transmission coefficients go to zero, while the number of channels goes to infinity: $M \rightarrow \infty$. The limits are taken in such a way that the sum over all transmission coefficients remains finite: $\sum_{c} T_{c}=T_{\text {sum }}$. In that case, the resonance widths stop fluctuating [37]. In the second case, we assume that all transmission coefficients are equal to one, while the number of channels is large but finite. We refer to that case as the semiclassical regime.

Absorptive limit In this case, the autocorrelation function can be computed in closed form (all integrals can be solved, see Appendix A), and one obtains:

$$
\hat{C}[\sigma]=\sigma_{\text {ind }}^{2} \mathrm{e}^{-T_{s} t}\left\{\left(1-\frac{1}{4} \sum_{a} \tau_{a}\right)^{2}\left[1-b_{2}(t)\right]+2+C_{0}\right\},
$$

where the constant $C_{0}$ depends on the statistical Fano parameters. Hence, in the absence of internal Fano interference, one can obtain a perfect exponential autocorrelation function by properly tuning $\sum_{a} \tau_{a} \rightarrow 4$.

Semiclassical regime For simplicity, we focus on the two extreme cases. In the first case, the decay is dominated by indirect processes, such that the autocorrelation function becomes:

$$
\hat{C}[\sigma](t)=4 \sigma_{\text {ind }}^{2} \mathcal{I}^{(M)}\left(\Delta_{0}^{2}+\Pi_{00}\right),
$$

while in the second case, it is dominated by direct processes. This results in

$$
\hat{C}[\sigma](t)=\sigma_{\text {ind }}^{2} \frac{\sum_{a, b}\left(\tau_{a}^{-} \tau_{b}^{+}+\tau_{a} \tau_{b}\right)}{8} \mathcal{I}^{(M)} \Pi_{11} .
$$

As can be seen from equation (A.1), the autocorrelation function is then proportional to the autocorrelation function of a diagonal S-matrix element (note that all transmission coefficients are equal to one). Thus, very large statistical Fano parameters (i.e. the dominance of the direct processes) result in a photo cross section which has the same statistical properties as a full scattering cross section $\sigma_{\text {tot }}(E)$ [36]. Below, we will thus compare $\hat{C}[\sigma](t)$ for indirect decay with $\hat{C}\left[\sigma_{\text {tot }}\right](t)$ for direct decay. Note that for large $M$, one expects the autocorrelation function in both cases to be dominated by an exponential decay with $\mathrm{e}^{-M t}[35,38,18]$. 


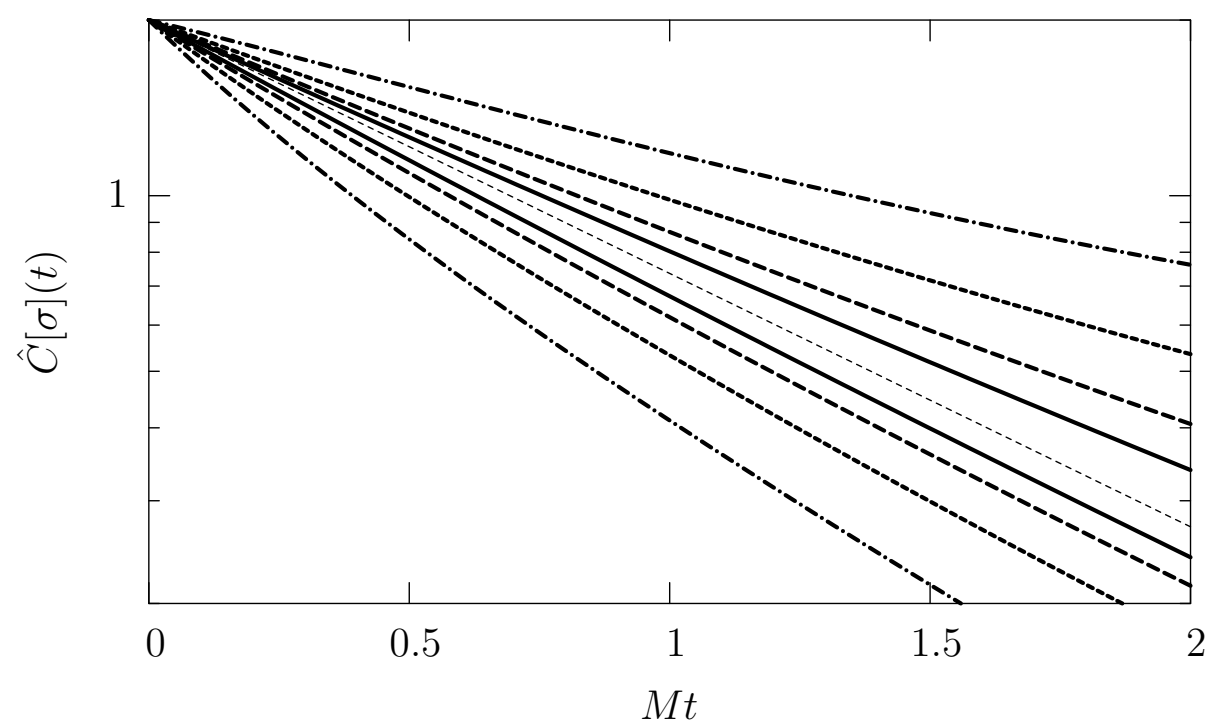

Figure 1. The autocorrelation function $\hat{C}[\sigma](t)$ versus $M t$, in the absence of direct processes (indirect cross section) compared to the same quantity where the direct processes dominate. The curves are computed numerically, on the basis of the VWZ integral, see Appendix A. All transmission coefficients are taken equal to one, and the number of channels is varied between $M=16$ (solid line), 8 (dashed line), 4 (dotted line), and 2 (dash-dotted line). The thin dotted line shows the exponential decay: $2 \exp (-M t)$.

In figure 1 we simply compare the resulting autocorrelation functions, varying the number of channels. A semilog plot is used, and on the ordinate $M t$ is given, such that differences to the exponential decay are easier recognized. The autocorrelation functions are normalized, such that $\hat{C}[\sigma](0)=\hat{C}\left[\sigma_{\text {tot }}\right](0)=2$. In the case of indirect decay, the autocorrelation function lies above the purely exponential decay (thin dotted line), approaching the exponential as $M$ increases. In the case of direct decay, the autocorrelation function approaches the exponential from below.

In figure 2 the same autocorrelation functions are shown, again. However, here, we divide by the classical expectation $\exp (-M t)$ and we plot the autocorrelation functions versus time $t$ (in units of the Heisenberg time). In this way, differences to the classical expectation are strongly enhanced. For indirect decay, the autocorrelation function has been computed semi-classically in [18], with the result: $\hat{C}[\sigma](t)=2(1+t) \mathrm{e}^{-M t}$. For the autocorrelation function of total scattering cross sections, a similar result is not available (to the best of the author's knowledge). One may however find a connection to the weak localization correction of mesoscopic transport [30], because the integral over the autocorrelation function in the time domain, gives the average value of the modulus squared of the corresponding S-matrix element [36]:

$$
\int_{0}^{\infty} \mathrm{d} t \hat{C}[\sigma](t)=\left\langle\left|S_{a a}\right|^{2}\right\rangle=\frac{2}{M+1} .
$$




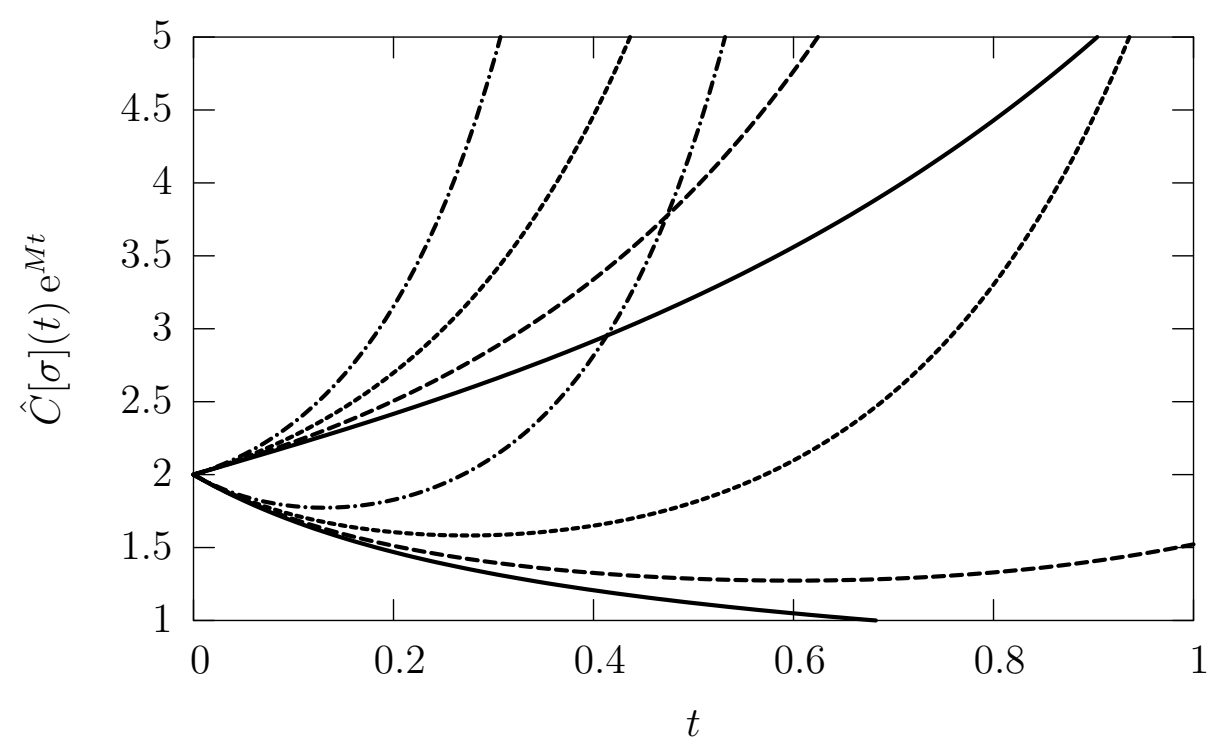

Figure 2. The same autocorrelation functions as in figure 1 , but divided by $\mathrm{e}^{-M t}$, and plotted versus $t$. Again, the number of channels is varied between $M=2$ (dash-dotted lines), $M=4$ (dotted lines), $M=8$ (dashed lines), and $M=16$ (solid lines). The autocorrelation functions for indirect decay have a positive slope at $t=0$, while in the opposite case, they have a negative slope.

\begin{tabular}{|r|c|c|c|}
\hline $\mathrm{M}$ & $\mathrm{a}$ & $\mathrm{b}$ & $\mathrm{c}$ \\
\hline 2 & $1.99934(54)$ & $0.076(48)$ & $0.1(15)$ \\
\hline 4 & $2.00018(35)$ & $1.991(30)$ & $3.67(87)$ \\
\hline 8 & $2.00029(44)$ & $5.987(37)$ & $5.0(10)$ \\
\hline 16 & $2.00057(21)$ & $13.953(17)$ & $8.74(49)$ \\
\hline
\end{tabular}

Table 1. The coefficients $a, b, c$ in equation (36) as obtained from a polynomial fit to the $\hat{C}[\sigma](t)$ as plotted in figure 2. The digits in brackets give the estimated error from the $\chi^{2}$-fit [32]. The first column gives the number of channels.

To study the behavior of the autocorrelation functions $\hat{C}[\sigma](t)$ (indirect decay) and $\hat{C}\left[\sigma_{\text {tot }}\right](t)$ more quantitatively, we perform a polynomial fit to the numerical results, as shown in figure 2. To this end we use the following general expression:

$$
\hat{C}[\sigma](t) \sim\left(2+a t+b t^{2}+c t^{3}+\ldots\right) \mathrm{e}^{-M t} .
$$

The results of the fits are given in the tables 1 and 2. We used a polynomial of fifth order for the fit, but the values for the higher order coefficients had too large errors to be of any use. On the basis of the values found, one may conjecture:

$$
\begin{aligned}
& \hat{C}[\sigma](t) \sim\left(2+2 t+(M-2) t^{2}\right) \mathrm{e}^{-M t} \\
& \hat{C}\left[\sigma_{\text {tot }}\right](t) \sim\left(2-4 t+(M+4) t^{2}\right) \mathrm{e}^{-M t} .
\end{aligned}
$$

In the case of indirect decay, we obtain agreement with [18] up to the linear term. Higher order semiclassical corrections to the indirect photo cross sections have not been 


\begin{tabular}{|r|c|c|c|}
\hline $\mathrm{M}$ & $\mathrm{a}$ & $\mathrm{b}$ & $\mathrm{c}$ \\
\hline 2 & $-4.00109(70)$ & $10.101(62)$ & $-27.1(19)$ \\
\hline 4 & $-4.00039(48)$ & $12.028(41)$ & $-32.5(12)$ \\
\hline 8 & $-3.99930(30)$ & $15.945(25)$ & $-46.21(72)$ \\
\hline 16 & $-3.99951(21)$ & $23.948(18)$ & $-77.58(49)$ \\
\hline
\end{tabular}

Table 2. The coefficients $a, b, c$ in equation (36) as obtained from a polynomial fit to $\hat{C}\left[\sigma_{\text {tot }}\right](t)$ as plotted in figure 2 . The digits in brackets give the estimated error from the $\chi^{2}$-fit [32]. The first column gives the number of channels.

considered, so far. In the second case, we may actually integrate equation (38) to recover the weak localization correction:

$$
\int_{0}^{\infty} \mathrm{d} t \hat{C}\left[\sigma_{\text {tot }}\right](t) \sim \frac{2}{M}-\frac{4}{M^{2}}+\frac{2}{M^{2}}+\ldots
$$

in agreement with an expansion of equation (35) in inverse powers of $M$. This may of course just be a coincidence. For a definite answer, one should try to integrate $\mathcal{I}^{(M)} \Pi_{11}$ in closed form.

\section{Conclusions}

This work has started out from a recent formulation of a statistical model for half collision processes $[22,23]$. The principal aim was to investigate the possibility to detect and quantify Fano interference in cases where the total photo cross section shows irregular behavior (that may be randomly fluctuating lineshapes and/or overlapping resonances). The analysis of the Fano-parameter distribution [26, 29] may be appropriate, as long as the resonances are well isolated. However, the cross section autocorrelation function, can always be analysed. Surprisingly, it provides practically the same information (statistical Fano parameters) as the analysis of the Fano-parameter distribution. This allowed us to investigate Fano interference in the regime of strongly overlapping resonances. There, Fano interference effects are much weaker (on the level of the weak localization correction).

One might have doubts, whether Fano interference can also be detected in practice, when many channels are strongly coupled to the interaction region. Given a dynamical system, the main question is, how much averaging over different initial conditions and/or different samples is necessary in order to obtain the desired information. To clarify that point, numerical simulations with the open kicked rotor $[39,40,41]$ are currently under investigation.

Fano interference has turned into a versatile tool in mesoscopic physics, where it is used to measure dephasing and decoherence times [26, 27]. So far, only single Fano resonances in single mode systems have been applied. It would be desirable, to be able to measure dephasing and decoherence times also in multi-mode systems, from irregular cross sections. As a first step in that direction, one should include dephasing and decoherence into the statistical model. 


\section{Acknowledgments}

Very fruitful discussions with D. F. Martinez, H. Schomerus and B. Mehlig are greatfully acknowledged.

\section{Appendix A. The Verbaarschot-Weidenmüller-Zirnbauer integral}

In the case that $H_{0}$ is taken from the GOE, the correlation function between two scattering matrix elements from equation (6) is given by the VWZ-integral [17]. Fouriertransformed into the time domain, it reads [36]:

$$
\hat{C}\left[S_{a b}, S_{c d}^{*}\right](t)=\mathcal{I}\left\{4 \delta_{a b} \delta_{c d} T_{a} T_{c} \Delta_{a} \Delta_{c}+2\left(\delta_{a c} \delta_{b d}+\delta_{a d} \delta_{b c}\right) T_{a} T_{b} \Pi_{a b}\right\} .
$$

Here, the $T_{a}$ are the transmission coefficients, and we used the following abbreviations:

$$
\begin{aligned}
\mathcal{I} & =\int_{\max (0, t-1)}^{t} \mathrm{~d} r \int_{0}^{r} \mathrm{~d} u \frac{(t-r)(r+1-t)}{(2 u+1)\left(t^{2}-r^{2}+x\right)^{2}} \prod_{e=1}^{M} \frac{1-T_{e}(t-r)}{\sqrt{1+2 T_{e} r+T_{e}^{2} x}} \\
\Delta_{a} & =\sqrt{1-T_{a}}\left(\frac{r+T_{a} x}{1+2 T_{a} r+T_{a}^{2} x}+\frac{t-r}{1-T_{a}(t-r)}\right) \quad x=u^{2} \frac{2 r+1}{2 u+1} \\
\Pi_{a b} & =\frac{T_{a} T_{b} x^{2}+\left[T_{a} T_{b} r+\left(T_{a}+T_{b}\right)(r+1)-1\right] x+(2 r+1) r}{\left(1+2 T_{a} r+T_{a}^{2} x\right)\left(1+2 T_{b} r+T_{b}^{2} x\right)}+\frac{(t-r)(r+1-t)}{\left[1-T_{a}(t-r)\right]\left[1-T_{b}(t-r)\right]} .
\end{aligned}
$$

Absorptive limit There, all transmission coefficients go to zero, while the number of channels goes to infinity: $M \rightarrow \infty$. Both limits are taken in such a way that $\sum_{c} T_{C}=T_{\text {sum }}$ remains finite. In that case, one find:

$$
\begin{aligned}
& \Delta_{a} \rightarrow \Delta_{0}=t \\
& \Pi_{a b} \rightarrow \Pi_{00}=(t-r)(r+1-t)-x+(2 r+1) r \\
& \mathcal{I} \quad \rightarrow \mathrm{e}^{-T_{s} t} \mathcal{I}^{(0)} \quad \mathcal{I}^{(0)}=\int_{\max (0, t-1)}^{t} \mathrm{~d} r \int_{0}^{r} \mathrm{~d} u \frac{(t-r)(r+1-t)}{(2 u+1)\left(t^{2}-r^{2}+x\right)^{2}} .
\end{aligned}
$$

The integrals $\mathcal{I}^{(0)}$ and $\mathcal{I}^{(0)} \Pi_{00}$ can be calculated in closed form [42]. This gives:

$$
\mathcal{I}^{(0)}=\frac{1-b_{2}(t)}{4 t^{2}} \quad \mathcal{I}^{(0)} \Pi_{00}=\frac{1}{2} .
$$

For the GOE spectrum, the two point form factor $b_{2}(t)$ is given by

$$
1-b_{2}(t)=2 t-t \ln (2 t+1)+\theta(t-1)[2-2 t+t \ln (2 t-1)] .
$$

This gives for the correlation function:

$\hat{C}\left[S_{a b}, S_{c d}^{*}\right](t)=\mathrm{e}^{-T_{s} t}\left[\delta_{a b} \delta_{c d} T_{a} T_{c}\left(1-b_{2}(t)\right)+\left(\delta_{a c} \delta_{b d}+\delta_{a d} \delta_{b c}\right) T_{a} T_{b}\right]$.

The semiclassical regime There, all transmission coefficients are set equal to one $\forall c: T_{c}=1$. Typically the number of channels $M$ is assumed to be large but finite. However, the following relations really hold for arbitrary $M$. In that case, one finds:

$$
\Delta_{a} \rightarrow\left\{\begin{array} { l l } 
{ \Delta _ { 0 } = t } & { : T _ { a } \rightarrow 0 } \\
{ \Delta _ { 1 } = 0 } & { : T _ { a } \rightarrow 1 }
\end{array} \quad \Pi _ { a b } \rightarrow \left\{\begin{array}{l}
\Pi_{00}: T_{a}, T_{b} \rightarrow 0 \\
\Pi_{01}: T_{a} \rightarrow 0, T_{b} \rightarrow 1 \\
\Pi_{11}: T_{a}, T_{b} \rightarrow 1
\end{array}\right.\right.
$$


where $\Pi_{00}$ is given in equation (A.3), and

$$
\Pi_{01}=t \quad \Pi_{11}=\frac{x+r}{1+2 r+x}+\frac{t-r}{1+r-t} .
$$

Finally,

$\mathcal{I} \rightarrow \mathcal{I}^{(M)}=\int_{\max (0, t-1)}^{t} \mathrm{~d} r \int_{0}^{r} \mathrm{~d} u \frac{(t-r)(r+1-t)}{(2 u+1)\left(t^{2}-r^{2}+x\right)^{2}}\left(\frac{1+r-t}{\sqrt{1+2 r+x}}\right)^{M}$.

The resulting integrals are computed numerically.

\section{References}

[1] Reid S A and Reisler H 1994 J. Chem. Phys. 1015683

[2] Dobbyn A J, Stumpf M, Keller H-M, Hase W L and Schinke R 1995 J. Chem. Phys. 1025867

[3] Dobbyn A J, von Dirke M, Schinke R and Fink R 1995 J. Chem. Phys. 1027070

[4] Peskin U, Miller W H and Reisler H 1995 J. Chem. Phys. 1028874

[5] Keller H-M, Floethmann H, Dobbyn A J, Schinke R, Werner H-J, Bauer C and Rosmus P 1996 J. Chem. Phys. 1054983

[6] Kirmse B, Abel B, Schwarzer D, Grebenshchikov S Yu and Schinke R 2000 J. Phys. Chem. A 10410398

[7] Grémaud B and Delande D 1997 Europhys. Lett. 40363

[8] Rost J-M 1998 Phys. Rep. 297271

[9] Garcia-Sucre M, Raseev G and Ross G C, editors 1991 half collision resonances in molecules: experimental and theoretical approaches American Institute of Physics, New York

[10] Schinke R 1993 Photodissociation dynamics Cambridge University Press, Cambridge

[11] Alhassid Y and Fyodorov Y V 1998 J. Phys. Chem. A 1029577

[12] Fyodorov Y V and Alhassid Y 1998 Phys. Rev. A 58 R3375

[13] Heller E J 1978 J. Chem. Phys. 682066

[14] Heller E J 1978 J. Chem. Phys. 683891

[15] Feshbach H 1958 Ann. Phys. (N.Y.) 5357

[16] Feshbach H 1962 Ann. Phys. (N.Y.) 19287

[17] Verbaarschot J J M, Weidenmüller H A and Zirnbauer M R 1985 Phys. Rep. 129367

[18] Agam O 1999 Phys. Rev. A 60 R2633

[19] Agam O 2000 Phys. Rev. E 611285

[20] Beutler H 1935 Z. Phys. 93177

[21] Fano U 1961 Phys. Rev. 1241866

[22] Alhassid Y, Fyodorov Y V, Gorin T, Ihra W and Mehlig B 2003 E-print: cond-mat/0309521

[23] Gorin T, Mehlig B and Ihra W 2004 J. Phys. A: Math. Gen. 37 L345

[24] Feshbach H 1992 theoretical nuclear physics: nuclear reactions Wiley, New York

[25] Sokolov V V, Rotter I, Savin D V and Müller M 1997 Phys. Rev. C 561044

[26] Clerk A A, Waintal X and Brouwer P W 2001 Phys. Rev. Lett. 864636

[27] Kobayashi K, Aikawa H, Sano A, Katsumoto S and Iye Y 2004 Phys. Rev. B 70035319

[28] Johnson A C, Marcus C M, Hanson M P and Gossard A C 2004 Phys. Rev. Lett. 93106803

[29] Ihra W 2002 Phys. Rev. A 66 020701(R)

[30] Mello P A and Baranger H U 1999 Waves Random Media 9105

[31] Savin D V, Fyodorov Y V and Sommers H-J 2005 E-print: nlin.CD/0506040

[32] Press W H, editor 1992 Numerical recipes in Fortran. Cambridge University Press

[33] Fyodorov Y V and Sommers H-J 1997 J. Math. Phys. 381918

[34] Guhr T, Müller-Groeling A and Weidenmüller H A 1998 Phys. Rep. 299189

[35] Engelbrecht C A and Weidenmüller H A 1973 Phys. Rev. C 8859

[36] Gorin T and Seligman T H 2002 Phys. Rev. E 65026214 
[37] Ericson T and Mayer-Kuckuk T 1966 Annu. Rev. Nucl. Sci. 16183

[38] Blümel R and Smilansky U 1988 Phys. Rev. Lett. 60477

[39] Fyodorov Y V and Sommers H-J 2000 JETP Lett. 72422

[40] Ossipov A, Kottos T and Geisel T 2003 Europhys. Lett. 62719

[41] Tworzydło J, Tajic A, Schomerus H and Beenakker C W J 2003 Phys. Rev. B 68115313

[42] Stöckmann H-J and Schäfer R 2004 New J. Phys. 6199 